(C) <2017>. This manuscript version is made available under the CC-BY-NC-ND 4.0 license http://creativecommons.org/licenses/by-nc-nd/4.0/

The definitive publisher version is available online at https://doi.org/ 10.1016/j.jsams.2017.09.597 


\title{
EEG-Based Assessment of Pilot Spatial Navigation on Aviation
} Simulator

\author{
Li-Wei Ko ${ }^{1,3}$, Peng-Wen Lai ${ }^{1}$, Yu-Kai Wang ${ }^{1,2}$, and Chin-Teng Lin ${ }^{1,2}$ \\ 1 Brain Research Center, National Chiao Tung University, Hsinchu, Taiwan \\ ${ }_{2}$ Centre for Artificial Intelligence, FEIT, University of Technology Sydney, Australia \\ 3 Departments of Biological Science and Technology, National Chiao Tung University, \\ Hsinchu, Taiwan
}

Iwko.brc@gmail.com,yukaiwang@cs.nctu.edu.tw,pw.lai5498@gmail.com,ctlin@mail .nctu.edu.tw

\begin{abstract}
Spatial navigation is a highly complex cognitive activity in which the pilots require to establish the spatial map in brain and plan patrols. This study used 32 channel wireless EEG caps (MINDO-32) (Yi-Hsin Yu et al., 2016) to records the pilot's brain activity during spatial navigation in M-2000 flight simulator of air force base Hsinchu Taiwan. In this study, the aim is to explore the neurophysiological differences between rookie and veterans during spatial navigation. In the experiment, we recorded the EEG data from 20 pilots (seven of rookies and 13 of veterans). The aviation simulator randomly starts 3 at five possible positions far away from the air force base (Aircraft 72 degree offsets), and then the pilots had to flight on the correct direction and directly back to Tactical Air Navigation (TACAN). In the behavior analysis, we decided all 40 trails into two groups: The first 20 trails and the last 20 trails. The average reaction times of first and last 20 trails show that the rookie pilots are longer than the veteran pilots. In this study, The D-values applied to evaluate the time difference between rookies and veterans. The D-values of the first 20 trails and the last 20 trails are 0.590 to 0.668 . In the EEG analy sis, independent component analysis (ICA), event-related spectral perturbation (ERSP) and coherence applied to investigate the significant changes of brain oscillations between rookie and veteran pilots during navigation training. In the parietal component, the delta $(1-3 \mathrm{~Hz})$ and theta $(4-7 \mathrm{~Hz})$ activations increase, meanwhile the alpha $(8-12 \mathrm{~Hz})$ oscillation decreases (Vann, S. D. et al., 2009). We used coherence method to compare the different wave's changes between the rookie subjects and veteran subjects of cruising. Compared with the veteran pilots, the theta coherence is significantly increased in the rookie pilots during cruising (P-Values $<0.0001,0.001,0.01$ ). According to the positive D-values, increased theta activations, and increased theta coherence, the rookie pilots should pay more attention during the cruising. This study shows the behavioral deference and brain dynamics between rookies and veterans during spatial navigation. In the near future, we may integrate MINDO-32 into flight simulator for assessing the pilots and improving the training performance during spatial navigation.
\end{abstract}

